\title{
UNIQUENESS AND CHARACTERIZATION OF THE MAXIMIZERS OF INTEGRAL FUNCTIONALS WITH CONSTRAINTS
}

\author{
CRISTINA DRAGHICI AND HICHEM HAJAIEJ
}

\begin{abstract}
In this paper we maximize a class of functionals under certain constraints. We find necessary and sufficient conditions for these maximizers to exist and be unique. Moreover, we characterize them and discuss the optimality of our results by constructing counterexamples when one of the hypotheses does not hold.
\end{abstract}

\section{INTRODUCTION}

Optimization problems appear in economics, mathematics, to name only a few areas. They refer to minimizing, respectively maximizing a certain functional under several constraints. These problems are also connected with rearrangements. Often times the minimizers, or maximizers satisfy certain symmetry and monotonicity conditions, i.e. increasing or decreasing.

For $(X, \mu)$ a measure space, and $F$ a cost function, one studies the following minimization problem:

$$
\inf _{\left(s_{1}, \ldots, s_{n}\right) \in \Gamma} \int_{X} F\left(t, s_{1}(t), \ldots, s_{n}(t)\right) d \mu(t),
$$

where $\Gamma$ is a certain set of constraints. This is known as Monge's optimal problem with several marginals. See, for example [4]. The infimum is attained, in special cases, when $s_{1}, \ldots, s_{n}$ are nondecreasing functions.

Motivation. In [13], the authors prove uniqueness of the minimum of the following variational problem:

$$
\begin{gathered}
\inf \left\{\frac{1}{2} \int|\nabla u|^{2}-\int G(|x|, u(x)) d x, u \in S_{c}\right\}, \\
S_{c}=\left\{u \in H^{1}\left(\mathbb{R}^{n}\right): \int u^{2}=c^{2}\right\} .
\end{gathered}
$$

Their problem is related to nonlinear optics, $x$ is the position relative to the optical axis, $G$ is determined by the index of refraction of the optical media and $c>0$ is a parameter related to the wave speed. The constraint ensures that the total intensity of the associated beam of light is finite. In [10], some properties of the minimum were shown: smoothness, exponential decay at infinity, shape. In our paper, we study the maximization problem:

$$
\sup \int G\left(|x|, u_{1}(x), \ldots, u_{n}(x)\right) d x
$$

under similar constraints. This permits a better understanding of the role of the gradient in (1.1) and its interactions with the integral functional (recall that $\inf _{u \in S_{c}} \int|\nabla u|^{2}=0$ ). Moreover, our maximization problem is strongly related to functional inequalities [6, 7] for which cases of equality 
were recenty established but neither characterization nor uniqueness results were shown. For a supermodular integrand $F$ [12], we have the generalised Hardy-Littlewood inequalities [2, 3, 5]:

$$
\int_{X} F\left(f_{1}(x), \ldots, f_{n}(x)\right) d \mu(x) \leq \int_{X} F\left(f_{1}^{*}(x), \ldots, f_{n}^{*}(x)\right) d \mu(x),
$$

where $f_{1}^{*}, \ldots, f_{n}^{*}$ are the symmetric decreasing rearrangements of $f_{1}, \ldots, f_{n}$, respectively, and $X$ is a particular metric space. For the definition of the symmetric decreasing rearrangement and $X$, see Section 2 .

In this paper we will find necessary and sufficient conditions to obtain existence and uniqueness of the maximizers of the following problem:

$$
\sup _{h_{1} \in \mathcal{C}_{1}, \ldots, h_{n} \in \mathcal{C}_{n}} \int F\left(f(x), h_{1}(x), \ldots, h_{n}(x)\right) d \mu(x) .
$$

We will show that this sup is in fact attained and that the functions $h_{1} \ldots, h_{n}$ must be symmetric decreasing under the assumption that the function $f$ is strictly symmetric decreasing.

The intial step in solving (1.3) is the case $n=1, F(x, y)=x y$ and $X=\mathbb{R}$. The classical Hardy-Littlewood inequality [9, 1] for two functions is:

$$
\int f(x) h(x) d x \leq \int f^{*}(x) h^{*}(x) d x .
$$

If we assume that $f$ is strictly symmetric decreasing, then the maximizer $g$ (satisfying certain mean and growth constraints) must also be symmetric decreasing, and it can be expressed in terms of a certain level set of $f$. The fact that $f$ is strictly symmetric decreasing implies that every ball is a level set of $f$.

Indeed, if we define

$$
\mathcal{C}_{1}=\left\{g: \mathbb{R} \rightarrow \mathbb{R} \mid 0 \leq g \leq 1, \int g(x) d x \leq 1\right\}
$$

then it is not hard to see that $\max _{h \in \mathcal{C}_{1}} \int_{\mathbb{R}} f(x) h(x) d x=\int_{\mathbb{R}} f(x) g(x) d x$, where $g=\chi_{A^{\sharp}}$ with $A^{\sharp}$ the ball centered at the origin with measure 1 .

This problem is closely related to the "bathtub principle" [11] which says the following: on a measure space $(X, \mu)$ with a function $f: X \rightarrow \mathbb{R}$ satisfying the condition $\mu(\{f<t\})<\infty, \forall t \in \mathbb{R}$ the minimization problem

$$
\inf _{0 \leq g \leq 1, \int_{X} g d \mu=1} \int_{X} f(x) g(x) d \mu(x)
$$

has a solution $g(x)=\chi_{\{f<s\}}(x)+c \chi_{\{f=s\}}(x)$, where $s=\sup \{t \mid \mu(\{f<t\}) \leq 1\}$ and $c \mu(\{f=s\})=$ $1-\mu(\{f<s\})$.

\section{Assumptions AND THE MAIN RESUlt}

Let $(X, \mu)$ be the Euclidean space $\mathbb{R}^{m}$ with the Lebesgue measure, the sphere $S^{m}$ with the canonical measure, or the hyperbolic space $\mathbb{H}^{m}$ with the canonical measure. We equip $X$ with the 
standard metric $d$, and we choose a special point $\mathbf{o} \in X$ to serve as the origin or the north pole. Given positive numbers $l_{i}, k_{i}$ (fixed), $i=1, \ldots, n$, we define the sets:

$$
\mathcal{C}_{i}=\left\{g: X \rightarrow \mathbb{R}_{+} \mid g \text { measurable }, 0 \leq g \leq k_{i}, \int_{X} g(x) d \mu(x) \leq l_{i}\right\},
$$

and $\mathcal{C}=\mathcal{C}_{1} \times \ldots \times \mathcal{C}_{n}$.

Consider some generic set $\mathcal{C}_{i}$ and a function $g \in \mathcal{C}_{i}$. We define the symmetric decreasing rearrangement of $g$, denoted by $g^{*}$, as follows:

$$
g^{*}(x)=\inf \{t \geq 0 \mid \mu(\{g>t\}) \leq \mu(B(d(x)))\}
$$

where $B(d(x))$ is the ball centered at $\mathbf{o}$ and with radius $d(x):=d(x, \mathbf{o})$.

By Chebysev's inequality applied to $g$, we have:

$$
\mu(\{g>t\}) \leq(1 / t) \int_{X} g(x) d \mu(x) \leq l_{i} / t, \quad \forall t>0,
$$

and thus, all level sets of $g$ have finite measure at every height $t>0$.

Clearly, $g^{*}$ is symmetric decreasing and its level sets have the same measure as the corresponding level sets of $g$.

Let $F$ be a supermodular function satisfying additional conditions. We assume that $f: X \rightarrow \mathbb{R}_{+}$ is a strictly symmetric decreasing function, and we study the optimization problem:

$$
\sup _{\left(g_{1}, \ldots, g_{n}\right) \in \mathcal{C}} \int_{X} F\left(f(x), g_{1}(x), \ldots, g_{n}(x)\right) d \mu(x) .
$$

We show that the supremum is actually achieved, and the problem admits a unique solution with $g_{1}, \ldots, g_{n}$ symmetric decreasing functions. The numbers $k_{i}, l_{i}$ must also satisfy the following condition: $l_{1} / k_{1}=l_{2} / k_{2}=\ldots=l_{n} / k_{n}$. Of course, we know that in general

$$
\int_{X} F\left(f(x), g_{1}(x), \ldots, g_{n}(x)\right) d x \leq \int_{X} F\left(f(x), g_{1}^{*}(x), \ldots, g_{n}^{*}(x)\right) d x
$$

and we expect the maximizers to be symmetric decreasing.

Definition 2.1. We say that $F: \mathbb{R}_{+}^{2} \rightarrow \mathbb{R}$ is supermodular if $F\left(x_{1}, y_{1}\right)+F\left(x_{2}, y_{2}\right) \geq F\left(x_{1}, y_{2}\right)+$ $F\left(x_{2}, y_{1}\right)$, whenever $x_{1} \leq x_{2}$ and $y_{1} \leq y_{2}$.

Fact 2.2. (a) If $F$ is supermodular and $F(x, 0)=0$ for every $x \in \mathbb{R}_{+}$, then $F\left(\cdot, y_{0}\right)$ is increasing for every fixed $y_{0}$.

(b) For every fixed $x_{2}>x_{1}$, the function $F\left(x_{2}, \cdot\right)-F\left(x_{1}, \cdot\right)$ is increasing.

Proof. (a) Fix $y_{0}$ and consider $x_{1}<x_{2}$. From the definition, $F\left(x_{1}, 0\right)+F\left(x_{2}, y_{0}\right) \geq F\left(x_{1}, y_{0}\right)+$ $F\left(x_{2}, 0\right)$, which implies $F\left(x_{2}, y_{0}\right) \geq F\left(x_{1}, y_{0}\right)$.

(b)Let $y_{1}<y_{2}$ and use the definition to get that $F\left(x_{2}, y_{2}\right)-F\left(x_{1}, y_{2}\right) \geq F\left(x_{2}, y_{1}\right)-F\left(x_{1}, y_{1}\right)$.

\section{The Hardy-LitTlewood inequality}

In what follows, we sometimes write $\int F\left(f, g_{1}, \ldots, g_{n}\right)$ for $\int_{X} F\left(f(x), g_{1}(x), \ldots, g_{n}(x)\right) d x$.

Recall that given positive numbers $l_{i}, k_{i}$, we defined the class

$$
\mathcal{C}_{i}=\left\{g: X \rightarrow \mathbb{R} \mid 0 \leq g \leq k_{i}, \int g \leq l_{i}\right\}
$$


We also use the following defintion: $\mathbb{R}_{+}=[0, \infty)$.

Proposition 3.1. Let $f: X \rightarrow \mathbb{R}_{+}$be strictly symmetric decreasing and let $F: \mathbb{R}_{+}^{2} \rightarrow \mathbb{R}$ be a Borel measurable supermodular function satisfying the following conditions:

(1) $F(s, 0)=0, \forall s \in \mathbb{R}_{+}$,

(2) $F(a, t b) \leq t F(a, b), \forall 0 \leq t \leq 1$, and $a, b \in \mathbb{R}_{+}$.

Let $\left\{f>t_{1}\right\}$ be a level set of $f$ with measure $l_{1} / k_{1}$ and $g=k_{1} \chi_{\left\{f>t_{1}\right\}}$. Then $g \in \mathcal{C}_{1}$ and:

$$
\int_{X} F(f(x), h(x)) d x \leq \int_{X} F(f(x), g(x)) d x, \quad \forall h \in \mathcal{C}_{1} .
$$

Moreover, the right-hand side is always finite.

Remark. Given $l_{1}$ and $k_{1}$ the conclusion of the proposition is still valid if we only suppose that $f$ is non-increasing and there exists $t_{1}$ such that $\mu\left(\left\{f>t_{1}\right\}\right)=l_{1} / k_{1}$.

Note 1. From condition (2) we have, if we write $F=F_{+}-F_{-}$, that

$$
F_{+}(f, h) \leq\left(h / k_{1}\right) F_{+}\left(f, k_{1}\right) \leq\left(h / k_{1}\right) F_{+}\left(f(0), k_{1}\right),
$$

from which we conclude that $F_{+}(f, h)$ is integrable for every $h \in \mathcal{C}_{1}$. However, $F_{-}$may not be integrable and then the integral $\int F(f, h)$ equals $-\infty$. Consider, for example, $F(x, y)=-\sqrt{y}$. The proof of the Proposition above holds when $F(f, h)$ is integrable. When this is not the case, the inequality in the Proposition holds trivially since the LHS is $-\infty$.

Note 2. Condition (1) in Proposition 3.1 can be weakened and replaced by an integral assumption. See Theorem 5.4 of [8].

Proof of Proposition 3.1. First, we show that $\int_{X} F(f(x), g(x)) d x=\int_{\left\{f>t_{1}\right\}} F\left(f(x), k_{1}\right) d x$ is finite. This follows from the following inequalities:

$$
F\left(t_{1}, k_{1}\right) \leq F\left(f(x), k_{1}\right) \leq F\left(f(0), k_{1}\right)
$$

and $\left|\left\{f>t_{1}\right\}\right|$ is finite.

Now we prove the main inequality. Let $h$ be a function in $\mathcal{C}_{1}$, that is $0 \leq h \leq k_{1}$ and $\int_{X} h(x) d x \leq$ $l_{1}$. If $\int F(f, h)$ equals $-\infty$ then there is nothing to prove. Otherwise, $F(f, h)$ is integrable (see Note 1 above) and we have the following:

$$
\begin{aligned}
\int F(f, h)= & \int_{\left\{f>t_{1}\right\}} F(f, h)+\int_{\left\{f \leq t_{1}\right\}} F(f, h) \\
& \leq \int_{\left\{f>t_{1}\right\}} F(f, h)+\int_{\left\{f \leq t_{1}\right\}} F\left(t_{1}, h\right)=\int_{\left\{f>t_{1}\right\}}\left[F(f, h)-F\left(t_{1}, h\right)\right]+\int F\left(t_{1}, h\right) \\
& \leq \int_{\left\{f>t_{1}\right\}}\left[F\left(f, k_{1}\right)-F\left(t_{1}, k_{1}\right)\right]+\left(1 / k_{1}\right) \int F\left(t_{1}, k_{1}\right) h \\
& =\int_{\left\{f>t_{1}\right\}} F\left(f, k_{1}\right)-F\left(t_{1}, k_{1}\right)\left(l_{1} / k_{1}\right)+F\left(t_{1}, k_{1}\right)\left(l_{1} / k_{1}\right)=\int F(f, g) .
\end{aligned}
$$

In the first inequality we used Fact 2.2 (a) ( $F$ is increasing in the first variable), and in the second inequality Fact 2.2 (b) and condition (2) in the Proposition. In the second equality we used the 
fact that both $\int_{\left\{f \leq t_{1}\right\}} F\left(t_{1}, h\right)$ and $\int_{\left\{f>t_{1}\right\}} F\left(t_{1}, h\right)$ are finite. It is clear from the first inequality that $\int_{\left\{f \leq t_{1}\right\}} F\left(t_{1}, h\right)>-\infty$. On the set $\left\{f>t_{1}\right\}$, and using that $F$ is supermodular, we have:

$$
\begin{aligned}
F\left(t_{1}, h\right) & \geq F(f, h)+F\left(t_{1}, k_{1}\right)-F\left(f, k_{1}\right) \\
& \geq F(f, h)+F\left(t_{1}, k_{1}\right)-F\left(f(0), k_{1}\right) .
\end{aligned}
$$

Integrating over the set of finite measure $\left\{f>t_{1}\right\}$ and using our assumption that $F(f, h)$ is integrable we obtain $\int_{\left\{f>t_{1}\right\}} F\left(t_{1}, h\right)>-\infty$.

Remark. We can also treat various problems using the same method as in Proposition 3.1. For example, we define a set

$$
\mathcal{D}_{1}=\left\{g: X \rightarrow \mathbb{R} \mid 0 \leq g \leq k_{i}, \int g^{p} \leq l_{i}\right\},
$$

with $0<p<\infty$. If we replace condition (2) with $F(a, t b) \leq t^{p} F(a, b)$, then we have the same inequality

$$
\int_{X} F(f, h) \leq \int_{X} F(f, g), \quad \forall h \in \mathcal{D}_{1}
$$

where $g=k_{1} \chi_{\left\{f>t_{1}\right\}}$ with $\left|\left\{f>t_{1}\right\}\right|=l_{1} / k_{1}^{p}$.

Proposition 3.2. Let $f: X \rightarrow \mathbb{R}_{+}$be strictly symmetric decreasing and let $F: \mathbb{R}_{+}^{2} \rightarrow \mathbb{R}$ be a Borel measurable supermodular function satisfying the following conditions:

(1) $\int|F(f, 0)|<\infty$,

(2) $F(a, t b)-F(a, 0) \leq t(F(a, b)-F(a, 0)), \forall 0 \leq t \leq 1$, and $a, b \in \mathbb{R}_{+}$.

Let $\left\{f>t_{1}\right\}$ be a level set of $f$ with measure $l_{1} / k_{1}$ and $g=k_{1} \chi_{\left\{f>t_{1}\right\}}$. Then $g \in \mathcal{C}_{1}$ and:

$$
\int_{X} F(f(x), h(x)) d x \leq \int_{X} F(f(x), g(x)) d x, \quad \forall h \in \mathcal{C}_{1} .
$$

Moreover, the right-hand side is finite.

Proof. Let $\tilde{F}\left(s_{0}, s_{1}\right)=F\left(s_{0}, s_{1}\right)-F\left(s_{0}, 0\right)$ and apply Proposition 3.1 to get

$$
\int F(f, h)-\int F(f, 0) \leq \int F(f, g)-\int F(f, 0),
$$

from which we conclude that $\int F(f, h) \leq \int F(f, g)$.

Again, the LHS of (3.1) may be $-\infty$ in some cases.

Note. Condition (2) in Propositions 3.1 and 3.2 is necessary. Consider $l_{1}=2, k_{1}=1$ in the definition of $\mathcal{C}_{1}$ and $F(x, y)=x \sqrt{y}$. Then $F$ does not satisfy (2) as it can be easily checked. Let $f(x)=(1 / 2) e^{-|x|}$, and $g(x)=\chi_{(-1,1)}(x), h(x)=e^{-|x|}$. Notice that both $g$ and $h$ belong to $\mathcal{C}_{1}$. We compute the following integrals:

$$
\begin{gathered}
\int F(f(x), g(x)) d x=\int_{0}^{1} e^{-x} d x=1-1 / e \text { and } \\
\int F(f(x), h(x)) d x=\int_{0}^{\infty} e^{-3 x / 2} d x=2 / 3,
\end{gathered}
$$

and we notice that $\int F(f(x), g(x)) d x<\int F(f(x), h(x)) d x$.

The next proposition involves three functions of which one is strictly symmetric decreasing. 
Proposition 3.3. Let $F: \mathbb{R}_{+}^{3} \rightarrow \mathbb{R}$ be a Borel measurable function satisfying the following conditions:

(1) $F\left(s_{0}, s_{1}, 0\right)=0$, for all $s_{0}, s_{1} \in \mathbb{R}_{+}$,

(2) $F(a, b, t c) \leq t F(a, b, c)$, for every $(a, b, c) \in \mathbb{R}_{+}^{3}$, and every $t \in(0,1)$,

(3) $F$ is increasing in the second variable,

(4) $F\left(\cdot, s_{1}, \cdot\right)$ is supermodular for every fixed $s_{1}$.

Let $f: X \rightarrow \mathbb{R}_{+}$be a strictly symmetric decreasing function. Given any positive numbers $l_{1}, l_{2}, k_{1}, k_{2}$ with $l_{1} / k_{1}=l_{2} / k_{2}$, we define functions $g_{1}=k_{1} \chi_{\left\{f>t_{1}\right\}}$ and $g_{2}=k_{2} \chi_{\left\{f>t_{1}\right\}}$, with $t_{1}$ chosen so that $\left|\left\{f>t_{1}\right\}\right|=l_{1} / k_{1}=l_{2} / k_{2}$.

Then, for any $h_{1} \in \mathcal{C}_{1}$ and $h_{2} \in \mathcal{C}_{2}$, the following inequality holds:

$$
\int F\left(f, h_{1}, h_{2}\right) \leq \int F\left(f, g_{1}, g_{2}\right),
$$

where the LHS may be $-\infty$. Moreover, the right-hand side is finite.

Note. $F$ is increasing in the first variable by Fact 2.2 .

Proof. Using condition (3) and splitting the integral into two sums, and using the fact that $f$ is increasing in the first variable, we obtain:

$$
\begin{aligned}
\int F\left(f, h_{1}, h_{2}\right) & \leq \int F\left(f, k_{1}, h_{2}\right)=\int_{\left\{f>t_{1}\right\}} F\left(f, k_{1}, h_{2}\right)+\int_{\left\{f \leq t_{1}\right\}} F\left(f, k_{1}, h_{2}\right) \\
& \leq \int_{\left\{f>t_{1}\right\}} F\left(f, k_{1}, h_{2}\right)+\int_{\left\{f \leq t_{1}\right\}} F\left(t_{1}, k_{1}, h_{2}\right) \\
& =\int_{\left\{f>t_{1}\right\}}\left[F\left(f, k_{1}, h_{2}\right)-F\left(t_{1}, k_{1}, h_{2}\right)\right]+\int F\left(t_{1}, k_{1}, h_{2}\right)
\end{aligned}
$$

In the first integral below, we use Fact 2.2 (b), and in the second integral we use condition (2) in the Proposition.

$$
\begin{aligned}
\text { RHS of }(\underline{3.2}) & \leq \int_{\left\{f>t_{1}\right\}}\left[F\left(f, k_{1}, k_{2}\right)-F\left(t_{1}, k_{1}, k_{2}\right)\right]+\int F\left(t_{1}, k_{1}, k_{2}\right) h_{2} / k_{2} \\
& =\int_{\left\{f>t_{1}\right\}} F\left(f, k_{1}, k_{2}\right)-F\left(t_{1}, k_{1}, k_{2}\right) l_{1} / k_{1}+F\left(t_{1}, k_{1}, k_{2}\right) l_{2} / k_{2} \\
& =\int F\left(f, g_{1}, g_{2}\right)
\end{aligned}
$$

We have shown that $\left(g_{1}, g_{2}\right)$ is a maximizer of the following problem:

$$
\max _{h_{1} \in \mathcal{C}_{1}, h_{2} \in \mathcal{C}_{2}} \int F\left(f(x), h_{1}(x), h_{2}(x)\right) d x .
$$

Definition 3.4. We say that $F: \mathbb{R}_{+}^{2} \rightarrow \mathbb{R}$ is strictly supermodular if whenever $x_{1}<x_{2}$ and $y_{1}<y_{2}$, strict inequality holds: $F\left(x_{1}, y_{1}\right)+F\left(x_{2}, y_{2}\right)>F\left(x_{1}, y_{2}\right)+F\left(x_{2}, y_{1}\right)$.

The folowing result can be proved in the same way as Fact 2.2 . 
Fact 3.5. (a) If $F$ is strictly supermodular and $F(x, 0)=0$ for every $x \in \mathbb{R}_{+}$, then $F\left(\cdot, y_{0}\right)$ is strictly increasing for every fixed $y_{0}>0$.

(b) For every fixed $x_{2}>x_{1}$, the function $F\left(x_{2}, \cdot\right)-F\left(x_{1}, \cdot\right)$ is strictly increasing.

Theorem 3.6. (Existence and uniqueness of maximizers) Let $F: \mathbb{R}_{+}^{3} \rightarrow \mathbb{R}$ be a Borel measurable function satisfying the following conditions:

(1) $F\left(s_{0}, s_{1}, 0\right)=0$, for all $s_{0}, s_{1} \in \mathbb{R}_{+}$,

(2) $F(a, b, t c) \leq t F(a, b, c)$, for every $(a, b, c) \in \mathbb{R}_{+}^{3}$, and every $t \in(0,1)$,

(3) $F(x, \cdot, y)$ is strictly increasing for every fixed $x, y>0$,

(4) $F\left(\cdot, s_{1}, \cdot\right)$ is strictly supermodular for every fixed $s_{1}$.

Let $f: X \rightarrow \mathbb{R}_{+}$be a strictly symmetric decreasing function. Given any positive numbers $l_{1}, l_{2}, k_{1}, k_{2}$ with $l_{1} / k_{1}=l_{2} / k_{2}$, we define functions $g_{1}=k_{1} \chi_{\left\{f>t_{1}\right\}}$ and $g_{2}=k_{2} \chi_{\left\{f>t_{1}\right\}}$, with $t_{1}$ chosen so that $\left|\left\{f>t_{1}\right\}\right|=l_{1} / k_{1}=l_{2} / k_{2}$.

Then, the maximizer $\left(g_{1}, g_{2}\right)$ in Proposition 3.3 is unique.

Note. The condition $l_{1} / k_{1}=l_{2} / k_{2}$ is necessary in order to obtain uniqueness of maximizers. Here is an example. Let $k_{1}=1, l_{1}=2$ and $k_{2}=2, l_{2}=1$. We define $f(x)=e^{-|x|}, F(x, y, z)=x y z$ and functions:

$$
\begin{array}{ll}
g_{1}=\chi_{(-1,1)} & h_{1}=\chi_{(-1 / 4,1 / 4)}+\chi_{(1,3 / 2)} \\
g_{2}=2 \chi_{(-1 / 4,1 / 4)} & h_{2}=2 \chi_{(-1 / 4,1 / 4)}
\end{array}
$$

Then $g_{1}, h_{1} \in \mathcal{C}_{1}, g_{2}, h_{2} \in \mathcal{C}_{2}$ and $\int F\left(f, h_{1}, h_{2}\right)=\int F\left(f, g_{1}, g_{2}\right)$, which shows that both $\left(g_{1}, g_{2}\right)$ and $\left(h_{1}, h_{2}\right)$ are maximizers.

Proof. Suppose $\left(\tilde{g_{1}}, \tilde{g_{2}}\right)$ is another maximizer couple and that $\tilde{g_{2}}<k_{2}$ on a set of positive measure of $\left\{f>t_{1}\right\}$. Using the same steps as in the proof of Proposition 3.3 and Fact 3.5 (b), we obtain:

$$
\begin{aligned}
\int F\left(f, \tilde{g_{1}}, \tilde{g_{2}}\right) & \leq \int_{\left\{f>t_{1}\right\}}\left[F\left(f, k_{1}, \tilde{g}_{2}\right)-F\left(t_{1}, k_{1}, \tilde{g_{2}}\right)\right]+\int F\left(t_{1}, k_{1}, \tilde{g}_{2}\right) \\
& <\int_{\left\{f>t_{1}\right\}}\left[F\left(f, k_{1}, k_{2}\right)-F\left(t_{1}, k_{1}, k_{2}\right)\right]+F\left(t_{1}, k_{1}, k_{2}\right) l_{2} / k_{2} \\
& =\int F\left(f, g_{1}, g_{2}\right),
\end{aligned}
$$

which gives a contradiction with the fact that $\left(\tilde{g_{1}}, \tilde{g_{2}}\right)$ is a maximizer. Thus, we must have $\tilde{g}_{2}=g_{2}$ a.e.

Next, assume $\tilde{g}_{1}<k_{1}$ on a set of positive measure of $\left\{f>t_{1}\right\}$. Then, using condition (3), we have:

$$
\int F\left(f, \tilde{g}_{1}, \tilde{g}_{2}\right)=\int_{\left\{f>t_{1}\right\}} F\left(f, \tilde{g}_{1}, k_{2}\right)<\int_{\left\{f>t_{1}\right\}} F\left(f, k_{1}, k_{2}\right)=\int F\left(f, g_{1}, g_{2}\right),
$$

and again we obtain a contradiction. This proves that the maximizing couple $\left(g_{1}, g_{2}\right)$ is unique.

Corollary 3.7. Let $f, g_{1}, g_{2}$ satisfy the conditions of Theorem 3.6. Let $F: \mathbb{R}_{+}^{3} \rightarrow \mathbb{R}$ be a Borel measurable function satisfying the following conditions:

(1) $\int\left|F\left(f, g_{1}, 0\right)\right|<\infty$, for every $g_{1} \in \mathcal{C}_{1}$,

(2) $F(a, b, t c)-F(a, b, 0) \leq t(F(a, b, c)-F(a, b, 0))$, for every $t \in(0,1)$, 
(3) $F(a, \cdot, c)-F(a, \cdot, 0)$ is increasing for any fixed $a, c$,

(4) $F(\cdot, b, \cdot)$ is supermodular for every fixed $b$.

Then:

$$
\int F\left(f, h_{1}, h_{2}\right)-\int F\left(f, h_{1}, 0\right) \leq \int F\left(f, g_{1}, g_{2}\right)-\int F\left(f, g_{1}, 0\right),
$$

$\forall h_{1} \in \mathcal{C}_{1}, h_{2} \in \mathcal{C}_{2}$. Under the additional assumptions that $F(a, t b, 0)-F(a, 0,0) \leq t(F(a, b, 0)-$ $F(a, 0,0)), \int|F(f, 0,0)|<\infty$, and $F(\cdot, \cdot, 0)$ is supermodular, we obtain that $\int F\left(f, h_{1}, 0\right) \leq$ $\int F\left(f, g_{1}, 0\right)$, which together with (3.3) yields:

$$
\int F\left(f, h_{1}, h_{2}\right) \leq \int F\left(f, g_{1}, g_{2}\right), \quad \forall h_{1} \in \mathcal{C}_{1}, h_{2} \in \mathcal{C}_{2} .
$$

Proof. To prove (3.3) we consider $\tilde{F}(x, y, z):=F(x, y, z)-F(x, y, 0)$ and we apply Proposition 3.3. The last inequality is deduced from the additional assumptions together with Proposition 3.2 . Indeed, the following inequality holds: $\int F\left(f, h_{1}, 0\right) \leq \int F\left(f, g_{1}, 0\right)$, for every $h_{1} \in \mathcal{C}_{1}$.

The next theorem is a generalization of the previous results to $n$ functions. The proof is very similar to the proof of Proposition 3.3, and will therefore be omitted.

Theorem 3.8. Let $F: \mathbb{R}_{+}^{n+1} \rightarrow \mathbb{R}$ be a Borel measurable function satisfying the following conditions:

(1) $F\left(s_{0}, s_{1}, \ldots, s_{n-1}, 0\right)=0$, for all $s_{0}, s_{1}, \ldots, s_{n-1} \in \mathbb{R}_{+}$,

(2) $F\left(a_{0}, a_{1}, \ldots, a_{n-1}, t a_{n}\right) \leq t F\left(a_{0}, a_{1}, \ldots, a_{n-1}, a_{n}\right)$, for every $t \in(0,1)$,

(3) $F$ is increasing in the $2,3, \ldots, n-1$ variables,

(4) $F\left(\cdot, a_{1}, \ldots, a_{n-1}, \cdot\right)$ is supermodular for every fixed $a_{1}, \ldots, a_{n-1}$.

Let $f: X \rightarrow \mathbb{R}_{+}$be a strictly symmetric decreasing function. Given any positive numbers $l_{1}, k_{1}, l_{2}, k_{2}, \ldots, l_{n}, k_{n}$ with $l_{1} / k_{1}=l_{2} / k_{2}=\ldots=l_{n} / k_{n}$, we define functions $g_{1}=k_{1} \chi_{\left\{f>t_{1}\right\}}$, $g_{2}=k_{2} \chi_{\left\{f>t_{1}\right\}}, \ldots, g_{n}=k_{n} \chi_{\left\{f>t_{1}\right\}}$ with $t_{1}$ chosen so that $\left|\left\{f>t_{1}\right\}\right|=l_{n} / k_{n}$.

Then, for any $h_{1} \in \mathcal{C}_{1}, h_{2} \in \mathcal{C}_{2}, \ldots, h_{n} \in \mathcal{C}_{n}$ the following inequality holds:

$$
\int F\left(f, h_{1}, \ldots, h_{n}\right) \leq \int F\left(f, g_{1}, \ldots, g_{n}\right),
$$

where the LHS may be $-\infty$. Moreover, the right-hand side is finite.

Note. Conditions $(1)-(4)$ in Theorem 3.8 correspond to the case $(1, n)$. The theorem remains valid if we restate these conditions for the case $(1, k), k=2, \ldots, n-1$. More precisely, here are the new conditions:

(1) $F\left(s_{0}, \ldots, s_{k-1}, 0, s_{k+1}, \ldots s_{n}\right)=0$,

(2) $F\left(a_{0}, \ldots, a_{k-1}, t a_{k}, a_{k+1}, \ldots, a_{n}\right) \leq t F\left(a_{0}, \ldots, a_{n}\right)$,

(3) $F$ is increasing in the $2, \ldots, k-1, k+1, \ldots, n$ variables,

(4) $F\left(\cdot, a_{1}, \ldots, a_{k-1}, \cdot, a_{k+1}, \ldots, a_{n}\right)$ is supermodular.

Theorem 3.9. (Existence and uniqueness of maximizers) Under the conditions of Theorem 3.8 and the additional conditions that $F$ is strictly increasing in the $2, \ldots, n-1$ variables and that $F\left(\cdot, a_{1}, \ldots, a_{n-1}, \cdot\right)$ is strictly supermodular for every fixed $a_{1}, \ldots, a_{n-1}$, we have uniqueness of the maximizers $g_{1}, \ldots, g_{n}$. 
The proof of the uniqueness result is similar to the proof of Theorem 3.6.

Note. Again, Theorem 3.9 remains valid if the hypotheses corresponding to the case $(1, n)$ are replaced by those corresponding to the case $(1, k)$.

Remark. The maximization problem mentioned in the introduction, $\sup \int G\left(|x|, u_{1}, \ldots, u_{n}\right)$ can be treated by defining

$$
F\left(s, u_{1}, \ldots, u_{n}\right):=G\left(-\ln s, u_{1}, \ldots, u_{n}\right)
$$

for $s \in(0,1]$ and applying the theorems involving $F$ and the function $f(x)=e^{-|x|}$. One can also state what conditions $G$ must satisfy in order for the theorems involving $F$ to apply.

\section{REFERENCES}

[1] C. Bennett and R. Sharpley. Interpolation of operators. Academic Press Inc., 1988.

[2] F. Brock. A general rearrangement inequality à la Hardy-Littlewood. J. Inequal. Appl., 5(4):309-320, 2000.

[3] A. Burchard and H. Hajaiej. Rearrangement inequalities for functionals with monotone integrands. J. Funct. Anal., 233(2):561-582, 2006.

[4] G. Carlier. On a class of multidimensional optimal transportation problems. J. Convex Anal., 10(2):517-529, 2003.

[5] J. A. Crowe, J. A. Zweibel, and P. C. Rosenbloom. Rearrangements of functions. J. Funct. Anal., 66(3):432-438, 1986.

[6] H. Hajaiej. Cases of equality and strict inequality in the extended Hardy-Littlewood inequalities. Proc. Roy. Soc. Edinburgh Sect. A, 135(3):643-661, 2005.

[7] H. Hajaiej. Extended Hardy-Littlewood inequalities and some applications. Trans. Amer. Math. Soc., 357(12):4885-4896 (electronic), 2005.

[8] H. Hajaiej and C. A. Stuart. Extensions of the Hardy-Littlewood inequalities for Schwarz symmetrization. Int. J. Math. Math. Sci., (57-60):3129-3150, 2004.

[9] G. H. Hardy, J. E. Littlewood, and G. Pólya. Inequalities. Cambridge Mathematical Library. Cambridge University Press, Cambridge, 1988. Reprint of the 1952 edition.

[10] H. Jeanjean and C. A. Stuart. Nonlinear eigenvalue problems having an unbounded branch of symmetric bound states. Adv. Differential Equations, 4(5):639-670, 1999.

[11] E. H. Lieb and M. Loss. Analysis, volume 14 of Graduate Studies in Mathematics. American Mathematical Society, Providence, RI, second edition, 2001.

[12] A. W. Marshall and I. Olkin. Inequalities: theory of majorization and its applications, volume 143 of Mathematics in Science and Engineering. Academic Press Inc. [Harcourt Brace Jovanovich Publishers], New York, 1979 .

[13] J. B. McLeod, C. A. Stuart, and W. C. Troy. Stability of standing waves for some nonlinear Schrödinger equations. Differential Integral Equations, 16(9):1025-1038, 2003.

Department of Mathematical Sciences, Aalborg University, Fr. Bajers Vej 7G, DK-9220 AalBORG EAST, DENMARK

E-mail address: cristina@math.aau.dk

Department of Mathematics, University of Zurich, Winterthurerstrasse 190, CH-8057 Zürich

E-mail address: hichem.hajaiej@gmail.com 